\title{
CORRECTION
}

\section{Author Correction: Regulation of sister chromatid cohesion by nuclear PD-L1}

Jia Yu (D), Bo Qin, Ann M. Moyer (D), Somaira Nowsheen (D), Xinyi Tu, Haidong Dong, Judy C. Boughey, Matthew P. Goetz, Richard Weinshilboum, Zhenkun Lou (iD) and Liewei Wang

Cell Research (2020) 30:823; https://doi.org/10.1038/s41422-020-0365-y

Correction to: Cell Research https://doi.org/10.1038/s41422-0200315-8, published online 29 April 2020

In the initial version of this article published online, there was a typo of the figure number in the discussion section. "Fig. $4 a$, b" should be "Fig. 5a, b". The correct information is as follows. This correction does not affect the description of the results or the conclusion of this work.

\section{DISCUSSION}

Data from Pd-I1 knockout mouse model show that PD-L1 is not essential for the maintenance of normal tissue homeostasis (Fig. $5 a, b)$. 